\title{
Detailed statistical results
}

\section{Non-specific gill score}

Significant terms are marked in red

\section{AVERAGE Non-specific gill score}

\begin{tabular}{|c|c|c|c|c|c|}
\hline \multicolumn{6}{|c|}{ Exposure day + 1 dphe } \\
\hline \multicolumn{6}{|c|}{ PERMANOVA table of results } \\
\hline Source & $\mathrm{df}$ & SS & MS & Pseudo-F & $\mathrm{P}($ perm $)$ \\
\hline Treatment & 1 & 1.5313 & 1.5313 & 5.8136 & 0.0245 \\
\hline Time & 1 & 0.28125 & 0.28125 & 1.0678 & 0.3216 \\
\hline Treatment $\mathrm{x}$ Time & 1 & 0.78125 & 0.78125 & 2.9661 & 0.098 \\
\hline Residuals & 28 & 7.375 & 0.26339 & & \\
\hline Total & 31 & 9.9688 & & & \\
\hline
\end{tabular}

\section{3 dphe onwards}

PERMANOVA table of results

\begin{tabular}{lrrrrrr} 
Source & df & SS & MS & Pseudo-F & P(perm) & P(MC) \\
\hline Treatment & 3 & 0.083333 & 0.027778 & 0.13333 & & 0.9409 \\
Time & 5 & 1.65 & 0.33 & 1.1061 & 0.3912 & \\
Tank (Treatment) & 4 & 0.83333 & 0.20833 & 0.90909 & 0.4734 & \\
Treatment x Time & 15 & 5.3167 & 0.35444 & 1.1881 & 0.3498 & \\
Tank (Treatment) x Time & 20 & 5.9667 & 0.29833 & 1.3018 & 0.1726 & \\
Residuals & 192 & 44 & 0.22917 & & & \\
Total & 239 & 57.85 & & &
\end{tabular}

Pooled terms

Tank (Treatment) x Time

PERMANOVA table of results

\begin{tabular}{lrrrrrr} 
Source & df & SS & MS & Pseudo-F & P(perm) & P(MC) \\
\hline Treatment & 3 & 0.083333 & 0.027778 & 0.13333 & & 0.9382 \\
Time & 5 & 1.65 & 0.33 & 1.4001 & 0.2168 & \\
Tank (Treatment) & 4 & 0.83333 & 0.20833 & 0.88392 & 0.4814 & \\
Treatment x Time & 15 & 5.3167 & 0.35444 & 1.5038 & 0.1068 & \\
Pooled & 212 & 49.967 & 0.23569 & & & \\
Total & 239 & 57.85 & & & &
\end{tabular}


Pooled terms

Tank (Treatment)

Tank (Treatment) x Time

PERMANOVA table of results

\begin{tabular}{lrrrrr} 
Source & df & SS & MS & Pseudo-F & P(perm) \\
\hline Treatment & 3 & 0.083333 & 0.027778 & 0.11811 & 0.949 \\
Time & 5 & 1.65 & 0.33 & 1.4031 & 0.2176 \\
Treatment x Time & 15 & 5.3167 & 0.35444 & 1.5071 & 0.0961 \\
Pooled & 216 & 50.8 & 0.23519 & & \\
Total & 239 & 57.85 & & &
\end{tabular}

No significant differences

\section{PREVALENCE for positive non-specific gill score}

Fishers Exact Test:

\begin{tabular}{ll} 
C-group vs. H-group & $p$ \\
\hline Exposure day & 0.343 \\
1 dphe & 0.029
\end{tabular}




\section{AGD gill score}

\section{Average AGD score}

\section{3 dphe onwards}

PERMANOVA table of results

\begin{tabular}{lrrrrrr} 
Source & df & SS & MS & Pseudo-F & P(perm) & P(MC) \\
\hline Treatment & 3 & 218.02 & 72.672 & 792.79 & & 0.0001 \\
Time & 5 & 97.233 & 19.447 & 60.456 & 0.0001 & \\
Tank (Treatment) & 4 & 0.36667 & 0.091667 & 0.47826 & 0.7633 & \\
Treatment x Time & 15 & 68.733 & 4.5822 & 14.245 & 0.0001 & \\
Tank (Treatment) x Time & 20 & 6.4333 & 0.32167 & 1.6783 & 0.0398 & \\
Residuals & 192 & 36.8 & 0.19167 & & & \\
Total & 239 & 427.58 & & & &
\end{tabular}

\section{PAIR-WISE TESTS}

Term 'Treatment x Time'

Within level ' 3 dphe' of factor 'time'

\begin{tabular}{lrr} 
Groups & $t$ & $\mathrm{P}(\mathrm{MC})$ \\
\hline Control, hydroids & Denominator is 0 \\
Control, P.per. & 6 & 0.0001 \\
Control, Hydroids + P.per. & 3 & 0.0076 \\
Hydroids, P.per. & 6 & 0.0001 \\
Hydroids, Hydroids + P.per. & 3 & 0.0079 \\
P.per., Hydro + P.per. & 1.4056 & 0.1722
\end{tabular}

Within level ' 7 dphe' of factor 'time'

\begin{tabular}{lrr} 
Groups & $\mathrm{t}$ & $\mathrm{P}(\mathrm{MC})$ \\
\hline Control, hydroids & Denominator is 0 \\
Control, P.per. & 1.964 & 0.0668 \\
Control, Hydroids + P.per. & 2.3333 & 0.0303 \\
Hydroids, P.per. & 1.964 & 0.0672 \\
Hydroids, Hydroids + P.per. & 2.3333 & 0.0338 \\
P.per., Hydro + P.per. & 1.1882 & 0.2503
\end{tabular}

Within level '14 dphe' of factor 'time'

\begin{tabular}{lc} 
Groups & $t \quad \mathrm{P}(\mathrm{MC})$ \\
\hline Control, hydroids & Denominator is 0 \\
Control, P.per. & $9 \quad 0.0001$ \\
Control, Hydroids + P.per. & Denominator is 0 \\
Hydroids, P.per. & $9 \quad 0.0001$ \\
Hydroids, Hydroids + P.per. & Denominator is 0 \\
P.per., Hydroids + P.per. & $1.5 \quad 0.1539$
\end{tabular}


Within level '21 dphe' of factor 'time'

\begin{tabular}{lrr} 
Groups & $\mathrm{t}$ & $\mathrm{P}(\mathrm{MC})$ \\
\hline Control, hydroids & 1 & 0.3212 \\
Control, P.per. & 15 & 0.0001 \\
Control, Hydroids + P.per. & 16.5 & 0.0001 \\
Hydroids, P.per. & 12.348 & 0.0001 \\
Hydroids, Hydroids + P.per. & 12.6 & 0.0001 \\
P.per., Hydroids + P.per. & 1.4056 & 0.1764
\end{tabular}

Within level '28 dphe' of factor 'time'

\begin{tabular}{lrr} 
Groups & $\mathrm{t}$ & $\mathrm{P}(\mathrm{MC})$ \\
\hline Control, hydroids & 1 & 0.3308 \\
Control, P.per. & 15.922 & 0.0001 \\
Control, Hydroids + P.per. & 11 & 0.0001 \\
Hydroids, P.per. & 13.056 & 0.0001 \\
Hydroids, Hydroids + P.per. & 9.3915 & 0.0001 \\
P.per., Hydroids + P.per. & 1.5492 & 0.1386
\end{tabular}

Within level '35 dphe' of factor 'time'

\begin{tabular}{lrr} 
Groups & $\mathrm{t}$ & $\mathrm{P}(\mathrm{MC})$ \\
\hline Control, hydroids & 5.0138 & 0.0001 \\
Control, P.per. & 17.335 & 0.0001 \\
Control, Hydroids + P.per. & 18.974 & 0.0001 \\
Hydroids, P.per. & 10.04 & 0.0001 \\
Hydroids, Hydroids + P.per. & 11.196 & 0.0001 \\
P.per., Hydroids + P.per. & 1 & 0.3332
\end{tabular}




\section{Thrombi score}

\section{Exposure day +1 dphe}

PERMANOVA table of results

\begin{tabular}{lrrrrr} 
Source & df & SS & MS & Pseudo-F & P(perm) \\
\hline Treatment & 1 & 7.0313 & 7.0313 & 28.636 & 0.0001 \\
Time & 1 & 9.0313 & 9.0313 & 36.782 & 0.0001 \\
Treatment x Time & 1 & 7.0313 & 7.0313 & 28.636 & 0.0001 \\
Residuals & 28 & 6.875 & 0.24554 & & \\
Total & 31 & 29.969 & & &
\end{tabular}

\section{PAIR-WISE TESTS}

Term 'Treatment x Time' for pairs of levels of factor 'Treatment'

Within level 'Exposure day' of factor 'Time'

Groups $\mathrm{t} P($ perm)

Control. hydroids Denominator is 0

Within level ' 1 dphe' of factor 'Time'

\begin{tabular}{lrr} 
Groups & $\mathrm{t}$ & $\mathrm{P}(\mathrm{MC})$ \\
\hline Control. hydroids & 5.3513 & 0.0001
\end{tabular}

\section{3 dphe onwards}

PERMANOVA table of results

\begin{tabular}{lrrrrrr} 
Source & df & SS & MS & Pseudo-F & P(perm) & P(MC) \\
\hline Treatment & 3 & 12.713 & 4.2375 & 19.189 & & 0,0092 \\
Time & 5 & 21.387 & 4.2775 & 15.794 & 0.0002 & \\
Tank (Treatment) & 4 & 0.88333 & 0.22083 & 0.99065 & 0.4057 & \\
Treatment x Time & 15 & 22.363 & 1.4908 & 5.5046 & 0.0003 & \\
Tank (Treatment) x Time & 20 & 5.4167 & 0.27083 & 1.215 & 0.2534 & \\
Residuals & 192 & 42.8 & 0.22292 & & & \\
Total & 239 & 105.56 & & & &
\end{tabular}

\section{Pooled terms}

Tank (Treatment) x Time

PERMANOVA table of results

\begin{tabular}{lrrrrrr} 
Source & df & SS & MS & Pseudo-F & P(perm) & P(MC) \\
\hline Treatment & 3 & 12.713 & 4.2375 & 19.189 & & 0,0072 \\
Time & 5 & 21.387 & 4.2775 & 18.807 & 0.0001 & \\
Tank (Treatment) & 4 & 0.88333 & 0.22083 & 0.97096 & 0.424 & \\
Treatment x Time & 15 & 22.363 & 1.4908 & 6.5549 & 0.0001 & \\
Pooled & 212 & 48.217 & 0.22744 & & & \\
Total & 239 & 105.56 & & & &
\end{tabular}




\section{Pooled terms}

Tank (Treatment)

Tank (Treatment) x Time

PERMANOVA table of results

\begin{tabular}{lrrrrr} 
Source & df & SS & MS & Pseudo-F & P(perm) \\
\hline Treatment & 3 & 12.713 & 4.2375 & 18.642 & 0.0001 \\
Time & 5 & 21.387 & 4.2775 & 18.818 & 0.0001 \\
Treatment x Time & 15 & 22.363 & 1.4908 & 6.5585 & 0.0001 \\
Pooled & 216 & 49.1 & 0.22731 & & \\
Total & 239 & 105.56 & & &
\end{tabular}

\section{PAIR-WISE TESTS}

Term 'Treatment x Time' for pairs of levels of factor 'Treatment'

Within level ' 3 dphe' of factor 'Time'

\begin{tabular}{lrr} 
Groups & $\mathrm{t}$ & $\mathrm{P}(\mathrm{MC})$ \\
\hline Control, Hydroids & 6.862 & 0.0001 \\
Control, P.per & 1.5 & 0.1493 \\
Control, Hydroids + P.per & 5.5816 & 0.0001 \\
Hydroids, P.per & 5.5317 & 0.0001 \\
Hydroids, Hydroids + P.per & 1.0366 & 0.3094 \\
P.Per, Hydroids + P.per & 4.3333 & 0.0004
\end{tabular}

Within level '7 dphe' of factor 'Time'

\begin{tabular}{lrr} 
Groups & $\mathrm{t}$ & $\mathrm{P}(\mathrm{MC})$ \\
\hline Control, Hydroids & 2.2778 & 0.0365 \\
Control, P.per & Negative & \\
Control, Hydroids + P.per & 3.8571 & 0.0011 \\
Hydroids, P.per & 2.2778 & 0.0334 \\
Hydroids, Hydroids + P.per & 0.55709 & 0.5883 \\
P.Per, Hydroids + P.per & 3.8571 & 0.0011
\end{tabular}

Within level '14 dphe' of factor 'Time'

\begin{tabular}{lrr} 
Groups & $\mathrm{t}$ & $\mathrm{P}(\mathrm{MC})$ \\
\hline Control, Hydroids & Denominator is 0 \\
Control, P.per & 1 & 0.3358 \\
Control, Hydroids + P.per & 1.4056 & 0.1802 \\
Hydroids, P.per & 1 & 0.328 \\
Hydroids, Hydroids + P.per & 1.4056 & 0.1766 \\
P.Per, Hydroids + P.per & 0.84853 & 0.4053
\end{tabular}

Within level ' 21 dphe' of factor 'Time'

\begin{tabular}{lrr} 
Groups & $\mathrm{t}$ & $\mathrm{P}(\mathrm{MC})$ \\
\hline Control, Hydroids & Negative & \\
Control, P.per & 1 & 0.3272 \\
Control, Hydroids + P.per & 1.2362 & 0.2297 \\
Hydroids, P.per & 1 & 0.3375 \\
Hydroids, Hydroids + P.per & 1.2362 & 0.2338 \\
P.Per, Hydroids + P.per & 1.8091 & 0.089
\end{tabular}


Within level '28 dphe' of factor 'Time'

\begin{tabular}{lrr} 
Groups & $\mathrm{t}$ & $\mathrm{P}(\mathrm{MC})$ \\
\hline Control, Hydroids & 0.84853 & 0.3997 \\
Control, P.per & Negative & \\
Control, Hydroids + P.per & $1.44 \mathrm{E}-08$ & 1 \\
Hydroids, P.per & 0.84853 & 0.3991 \\
Hydroids, Hydroids + P.per & 0.84853 & 0.4084 \\
P.Per, Hydroids + P.per & Negative &
\end{tabular}

Within level '35 dphe' of factor 'Time'

\begin{tabular}{llrr} 
Groups & & $\mathrm{t}$ & $\mathrm{P}(\mathrm{MC})$ \\
\hline Control, Hydroids & & 1 & 0.3215 \\
Control, P.per & Negative & & \\
Control, Hydroids + P.per & Negative & & \\
Hydroids, P.per & & 1 & 0.3387 \\
Hydroids, Hydroids + P.per & & 1 & 0.3295 \\
P.Per, Hydroids + P.per & Negative & &
\end{tabular}

\section{PREVALENCE for positive Thrombi score}

Fishers Exact Test:

\begin{tabular}{lr} 
C-group vs. H-group & $\mathrm{p}$ \\
\hline Exposure day & 0.342 \\
1 dphe & 0.01
\end{tabular}




\section{Hyperplasia score}

\section{AVERAGE Hyperplasia score}

\section{Exposure day +1 dphe}

PERMANOVA table of results

\begin{tabular}{lrrrrr} 
Source & df & SS & MS & Pseudo-F & P(perm) \\
\hline Treatment & 1 & 1.5313 & 1.5313 & 3.0901 & 0.0946 \\
Time & 1 & 0.03125 & 0.03125 & 0.063063 & 0.8315 \\
Treatment x Time & 1 & 1.5313 & 1.5313 & 3.0901 & 0.0906 \\
Residuals & 28 & 13.875 & 0.49554 & & \\
Total & 31 & 16.969 & & &
\end{tabular}

No significant differences

\section{3 dphe onwards}

PERMANOVA table of results

\begin{tabular}{lrrrrrr} 
Source & df & SS & MS & Pseudo-F & $P($ perm $)$ & $P(M C)$ \\
\hline Treatment & 3 & 14.733 & 4.9111 & 147.33 & & 0,0002 \\
Time & 5 & 24.583 & 4.9167 & 8.1492 & 0.0006 & \\
Tank (Treatment) & 4 & 0.13333 & 0.033333 & 0.048193 & 0.9961 & \\
Treatment x Time & 15 & 29.617 & 1.9744 & 3.2726 & 0.0089 & \\
Tank (Treatment) x Time & 20 & 12.067 & 0.60333 & 0.87229 & 0.6216 & \\
Residuals & 192 & 132.8 & 0.69167 & & & \\
Total & 239 & 213.93 & & & &
\end{tabular}

Pooled terms

Tank (Treatment) x Time

PERMANOVA table of results

\begin{tabular}{lrrrrrr} 
Source & df & SS & MS & Pseudo-F & $P($ perm $)$ & $P(M C)$ \\
\hline Treatment & 3 & 14.733 & 4.9111 & 147.33 & & 0,0002 \\
Time & 5 & 24.583 & 4.9167 & 7.1951 & 0.0001 & \\
Tank (Treatment) & 4 & 0.13333 & 0.033333 & 0.04878 & 0.995 & \\
Treatment x Time & 15 & 29.617 & 1.9744 & 2.8894 & 0.0006 & \\
Residuals & 212 & 144.87 & 0.68333 & & & \\
Total & 239 & 213.93 & & & &
\end{tabular}

Pooled terms

Tank (Treatment)

Tank (Treatment) x Time

PERMANOVA table of results

\begin{tabular}{lrrrrr} 
Source & df & SS & MS & Pseudo-F & P(perm) \\
\hline Treatment & 3 & 14.733 & 4.9111 & 7.3159 & 0.0002 \\
Time & 5 & 24.583 & 4.9167 & 7.3241 & 0.0001 \\
Treatment x Time & 15 & 29.617 & 1.9744 & 2.9412 & 0.0004 \\
Pooled & 216 & 145 & 0.6713 & & \\
Total & 239 & 213.93 & & &
\end{tabular}




\section{PAIR-WISE TESTS}

Term 'Treatment $\mathrm{x}$ Time' for pairs of levels of factor

Within level '3 dphe' of factor 'Time'

\begin{tabular}{lrr} 
Groups & $\mathrm{t}$ & $\mathrm{P}(\mathrm{MC})$ \\
\hline Control, Hydroids & 0.67082 & 0.523 \\
Control, P.per & $3.82 \mathrm{E}-08$ & 1 \\
Control, Hydroids + P.per & 1.3416 & 0.1978 \\
Hydroids, P.per & 0.47434 & 0.6312 \\
Hydroids, Hydroids + P.per & 0.6396 & 0.5316 \\
P.Per, Hydroids + P.per & 0.94868 & 0.3548
\end{tabular}

Within level '7 dphe' of factor 'Time'

\begin{tabular}{lrr} 
Groups & $\mathrm{t}$ & $\mathrm{P}(\mathrm{MC})$ \\
\hline Control, Hydroids & 0.25631 & 0.8027 \\
Control, P.per & $1.47 \mathrm{E}-08$ & 1 \\
Control, Hydroids + P.per & 0.70065 & 0.489 \\
Hydroids, P.per & 0.3046 & 0.7621 \\
Hydroids, Hydroids + P.per & 1.1442 & 0.2634 \\
P.Per, Hydroids + P.per & 0.80498 & 0.4275
\end{tabular}

Within level '14 dphe' of factor 'Time'

\begin{tabular}{lrr} 
Groups & $\mathrm{t}$ & $\mathrm{P}(\mathrm{MC})$ \\
\hline Control, Hydroids & 0.46291 & 0.6541 \\
Control, P.per & 0.43759 & 0.6688 \\
Control, Hydroids + P.per & 1.5213 & 0.1473 \\
Hydroids, P.per & $1.78 \mathrm{E}-08$ & 1 \\
Hydroids, Hydroids + P.per & 2.4 & 0.0262 \\
P.Per, Hydroids + P.per & 2.1909 & 0.0365
\end{tabular}

Within level '21 dphe' of factor 'Time'

\begin{tabular}{lrr} 
Groups & $\mathrm{t}$ & $\mathrm{P}(\mathrm{MC})$ \\
\hline Control, Hydroids & 0.91499 & 0.3726 \\
Control, P.per & 1.6348 & 0.1214 \\
Control, Hydroids + P.per & 0.3873 & 0.7035 \\
Hydroids, P.per & 4.0931 & 0.0009 \\
Hydroids, Hydroids + P.per & 1.5213 & 0.1438 \\
P.Per, Hydroids + P.per & 1.3007 & 0.2061
\end{tabular}

Within level '28 dphe' of factor 'Time'

\begin{tabular}{lrr} 
Groups & $\mathrm{t}$ & $\mathrm{P}(\mathrm{MC})$ \\
\hline Control, Hydroids & $1.89 \mathrm{E}-08$ & 1 \\
Control, P.per & 2.4495 & 0.0247 \\
Control, Hydroids + P.per & 2.3333 & 0.0319 \\
Hydroids, P.per & 2.2283 & 0.0411 \\
Hydroids, Hydroids + P.per & 2.0896 & 0.0507 \\
P.Per, Hydroids + P.per & 0.39736 & 0.6942
\end{tabular}


Within level '35 dphe' of factor 'Time'

\begin{tabular}{lrr} 
Groups & $\mathrm{t}$ & $\mathrm{P}(\mathrm{MC})$ \\
\hline Control, Hydroids & 0.84665 & 0.4079 \\
Control, P.per & 6.3258 & 0.0001 \\
Control, Hydroids + P.per & 6.5485 & 0.0001 \\
Hydroids, P.per & 4.4272 & 0.0006 \\
Hydroids, Hydroids + P.per & 4.6663 & 0.0002 \\
P.Per, Hydroids + P.per & 0.44721 & 0.6585
\end{tabular}

\section{PREVALENCE for positive Hyperplasia score}

Fishers Exact Test:

\begin{tabular}{lr} 
C-group vs. H-group & $p$ \\
\hline Exposure day & 0.342 \\
1 dphe & 0.01
\end{tabular}




\section{AGD lesion score}

\section{AVERAGE AGD lesion score}

\section{3 dphe onwards}

PERMANOVA table of results

\begin{tabular}{lrrrrrr} 
Source & df & SS & MS & Pseudo-F & $P($ perm $)$ & $P(M C)$ \\
\hline Treatment & 3 & 27.546 & 9.1819 & 48.97 & & 0.0013 \\
Time & 5 & 39.237 & 7.8475 & 101.26 & 0.0001 & \\
Tank (Treatment) & 4 & 0.75 & 0.1875 & 1.9565 & 0.101 \\
Treatment x Time & 15 & 40.179 & 2.6786 & 34.563 & 0.0001 \\
Tank (Treatment) x Time & 20 & 1.55 & 0.0775 & 0.8087 & 0.709 \\
Residuals & 192 & 18.4 & 0.095833 & & & \\
Total & 239 & 127.66 & & &
\end{tabular}

\section{Pooled terms}

Tank (Treatment) x Time

PERMANOVA table of results

\begin{tabular}{lrrrrrr} 
Source & df & SS & MS & Pseudo-F & P(perm) & $P(M C)$ \\
\hline Treatment & 3 & 27.546 & 9.1819 & 48.97 & & 0.002 \\
Time & 5 & 39.237 & 7.8475 & 83.392 & 0.0001 & \\
Tank (Treatment) & 4 & 0.75 & 0.1875 & 1.9925 & 0.0979 & \\
Treatment x Time & 15 & 40.179 & 2.6786 & 28.464 & 0.0001 & \\
Residuals & 212 & 19.95 & 0.094104 & & & \\
Total & 239 & 127.66 & & & &
\end{tabular}

\section{PAIR-WISE TESTS}

Term 'Treatment $x$ Time' for pairs of levels of factor 'Treatment'

Too little data available for 3, 7 and 14 dphe. Treatment groups "Control" and "Hydroids" had only 0 values

Within level '21 dphe' of factor 'Time'

\begin{tabular}{lcrr} 
Groups & $\mathrm{t}$ & $\mathrm{P}($ perm $)$ & $\mathrm{P}(\mathrm{MC})$ \\
\hline Control, Hydroids & Denominator is 0 & & \\
Control, P.per & 5 & 0,3368 & 0,0394 \\
Control, Hydroids + P.per & 1 & 1 & 0,427 \\
Hydroids, P.per & 5 & 0,3386 & 0,0379 \\
Hydroids, Hydroids + P.per & 1 & 1 & 0,4192 \\
P.Per, Hydroids + P.per & 1,3416 & 0,6626 & 0,3141
\end{tabular}


Within level '28 dphe' of factor 'Time'

\begin{tabular}{lcrr} 
Groups & $\mathrm{t}$ & $\mathrm{P}($ perm $)$ & $\mathrm{P}(\mathrm{MC})$ \\
\hline Control, Hydroids & Denominator is 0 & & \\
Control, P.per & Denominator is 0 & & \\
Control, Hydroids + P.per & 4,3333 & 0,3234 & 0,0553 \\
Hydroids, P.per & Denominator is 0 & & \\
Hydroids, Hydroids + P.per & 4,3333 & 0,3358 & 0,0502 \\
P.Per, Hydroids + P.per & 1 & 1 & 0,4308
\end{tabular}

Within level '35 dphe' of factor 'Time'

\begin{tabular}{lcrr} 
Groups & $\mathrm{t}$ & $\mathrm{P}($ perm $)$ & $\mathrm{P}(\mathrm{MC})$ \\
\hline Control, Hydroids & Denominator is 0 & & \\
Control, P.per & Denominator is 0 & & \\
Control, Hydroids + P.per & 11 & 0,3277 & 0,0092 \\
Hydroids, P.per & Denominator is 0 & & \\
Hydroids, Hydroids + P.per & 11 & 0,3245 & 0,0076 \\
P.Per, Hydroids + P.per & 1 & 1 & 0,4276
\end{tabular}

\section{PREVALENCE for positive AGD lesion score}

Fishers Exact Test*:

\begin{tabular}{lr} 
PP- vs. H+PP group & \multicolumn{1}{l}{$p$} \\
\hline 21 dphe & 0.626 \\
28 dphe & 0.1
\end{tabular}

*Conducted for days with sufficient data 